\title{
Body Image Disorders: Comparison between Unilateral Hemisphere Damage and Schizophrenia
}

\section{J. CUTTING}

Bethlem Royal Hospital, Monks Orchard Road, Beckenham, Kent, BR3 3BX, UK

\begin{abstract}
The records of a consecutive series of 100 schizophrenics were examined for any mention of body image disorders. These were present in 45 patients, the most common varieties being anomalous bodily experiences. The pattern of these was then compared with the sorts of body image disorders which occur in patients with left hemisphere lesions and in those with right hemisphere lesions. Schizophrenic body image disorders resembled those seen with right-sided lesions much more than they did those occurring following left-sided damage. This result is discussed in relationship to theories of hemisphere imbalance in schizophrenia.
\end{abstract}

\section{Introduction}

The term "body image disorder" covers all those phenomena where a subject experiences his or her body as different in some way or behaves as if it were different from before. The term was originally coined by Schilder (1935) as an elaboration of Head and Holmes' (1911) postural body schema. The terminology and classification of the various types of body image disorder is confusing. I (Cutting, 1990) have suggested five main varieties (Table 1).

First, there are disorders involving impaired identification of body parts. These include autotopagnosia, where a subject is no longer able to point to body parts on request, and finger agnosia, where this impaired identification is confined to fingers. Right-left disorientation also falls into this first category of body image disorders.

Secondly, there are disorders in which the main pathological element is bodily unawareness. Here, a subject fails to notice the existence of part of the body. In hemiasomatognosia, he or she neglects an entire half of the body. In anosognosia, a paralyzed limb is ignored and actively denied. In somatoparaphrenia, there is delusional elaboration that the paralyzed limb belongs to someone else.

Thirdly, there are what can best be described as anomalous bodily experiences. These cover all forms of distortion of some quality of bodily experience, e.g. alterations in sizes of a body part, displacements of body parts in space, or feelings of non-belongingness.

Fourthly, there are false body experiences. These are hallucinations of nonexistent bodily stimuli, as distinct from anomalous experiences in which an existing body part or real sensation is experienced differently from usual. 
TABLE 1. Suggested classification of body image disorders

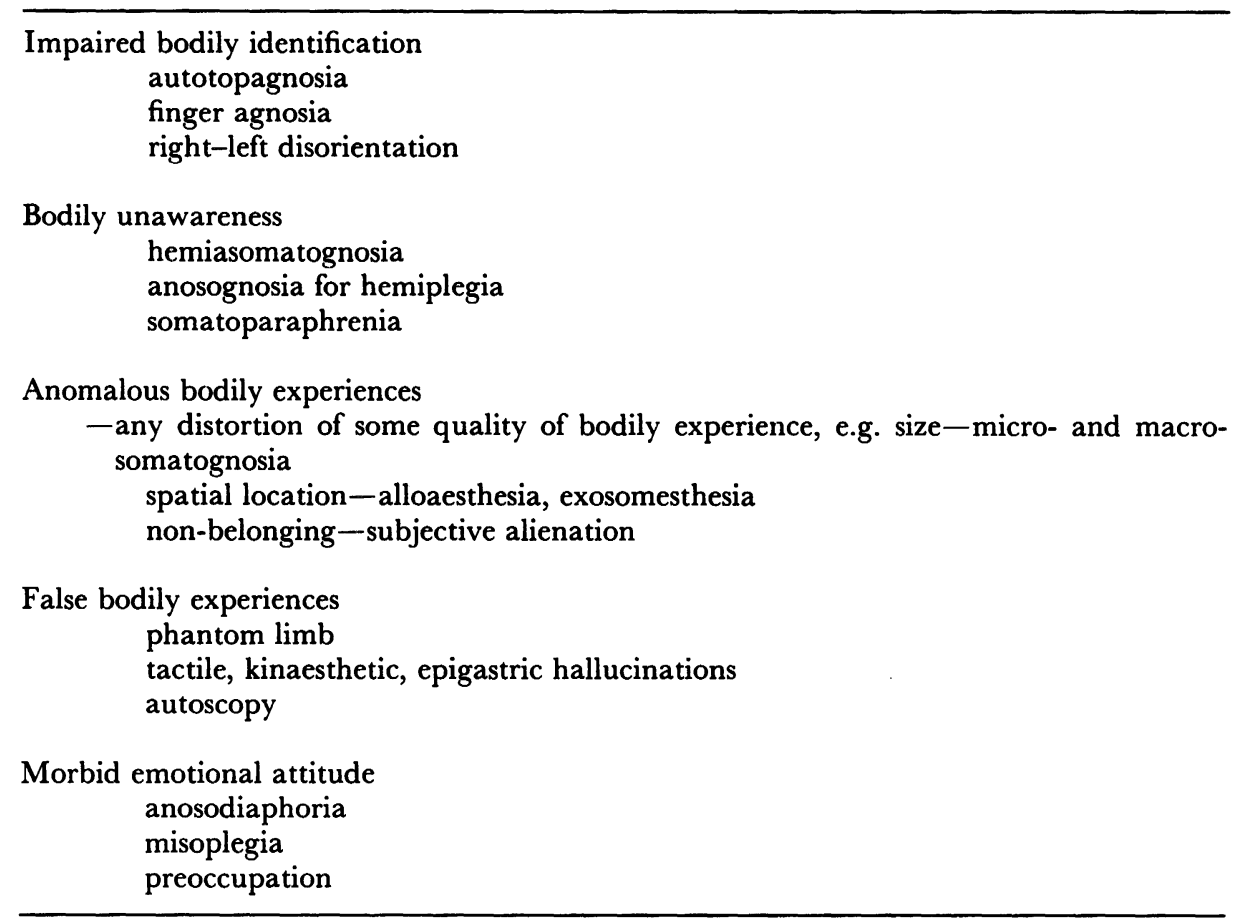

Phantom limb and autoscopic hallucinations (hallucinations of self) are false bodily experiences.

Finally, a subject may develop a distinctly morbid emotional attitude towards a body part, usually a limb. Babinski (1914) noted that some hemiplegics adopted a euphoric attitude towards a disability, with a tendency to belittle the consequences-anosodiaphoria. Critchley (1979) noticed that other hemiplegics took the opposite view of their conditionhating their disability with a fury out of keeping with its real significancemisoplegia. Van Bogaert (1934) also drew attention to a morbid preoccupation with disability, which again seemed to be more extreme than the situation warranted.

The classification I have suggested has the merit, in my view, that it divides up body image disorders according to psychological principles.

The first group-impaired identification of body parts-have in common a disorder of what can be termed categorical representation. It is not merely a selective aphasia for names of body parts, as has been suggested by some investigators, because patients with autotopagnosia (De Renzi and Scotti, 1970; Ogden, 1985) can accurately name their own body parts if the examiner touches them, and some patients (De Renzi and Scotti, 1970) demonstrate a wider problem in appreciating categorical relationships in that they cannot point to parts of other multipart objects on request (e.g. 
handlebars and brakes on a bicycle). The essential deficit involves what Stengel (1944) referred to as an "inability to locate a part of a multiplicity of objects forming an organized whole" or, in the words of De Renzi and Scotti (1970), an "inability to analyze whole into parts".

The second group of disorders-bodily unawareness--share a disturbance of attention. Part of the body is literally blotted out of awareness.

Anomalous bodily experiences, which are the main focus of this article, are perceptual disorders, in which the quality of the experience of an existing body part or stimulus is altered.

False bodily experiences are also perceptual disorders, but in this case a nonexistent body part or stimulus is hallucinated.

The psychological basis of a morbid emotional attitude to a body part is not clear, and there may be different mechanisms involved in different varieties. Nevertheless, they share the feature of having no consistent cognitive deficit. A patient of Gooddy and Reinhold (1953), for example, following a left-sided cerebrovascular accident, had no disability at all in his right limbs but complained bitterly that they "got in the way". A patient of Critchley (1951) with left-sided temporal lobe epilepsy complained that her right hand, during an aura, looked ugly and spiteful.

The classification suggested above also has the merit that certainly two and possibly three of the five groups have a consistent lateralization of the brain damage which gives rise to them.

Impaired identification of body parts is almost invariably a consequence of left hemisphere damage. Lunn (1948), for example, reviewed all anatomically verified cases to that date of Gerstmann's syndrome, of which finger agnosia is one of the tetrad of deficits, and found not one instance of a righthander with a right hemisphere lesion. Hecaen and Sauguet (1971) obtained an incidence of finger agnosia of $19 \%$ among 293 right-handers with a left hemisphere lesion and only $1 \%$ among 194 right-handers with a right hemisphere lesion.

The second group of disorders, on the other hand, are intimately related to right hemisphere damage. There were 31 instances of anosognosia, for example, among 100 patients with a recent onset of acute hemiplegia (Cutting, 1978). Twenty-eight of these had a right hemisphere lesion and denied a left hemiplegia-incidence of $58 \%$ among patients with right hemisphere damage. Only three had left hemisphere damage-incidence of $6 \%$ among patients with left hemisphere damage.

There is a possible link too between a certain type of morbid emotional attitude towards a body part and a lateralized focus of brain damage. Most instances of misoplegia or morbid preoccupation have followed a left hemisphere lesion. In my study of 100 recently incurred hemiplegias there were seven instances of misoplegia - five with a left-sided lesion $(23 \%$ of right hemiplegics who were free enough of aphasia to be interviewed) and only two with a right-sided lesion (4\% of interviewable left hemiplegics).

What has not been clear from the literature so far is whether anomalous and false bodily experiences, the remaining two groups of body image disorders in my classification, have any lateralized bias in the responsible 
brain damage. One of the purposes of this article is to examine this point, with particular respect to anomalous bodily experiences.

The second purpose of the article is to draw attention to the high rate of body image disorders in schizophrenia, a point which has been ignored by most commentators on the condition's phenomenological aspects. Only Angyal (1936), Lukianowicz (1967) and Taylor and Fleminger (1981) have, to my knowledge, addressed the issue in any systematic way in the Englishlanguage literature.

Angyal described the body image disorders which he encountered in 15 schizophrenics, one of these in exceptional detail. Virtually all the phenomena which he elicited were anomalous bodily experiences:

"Head and neck do not connect ... space between the chest and the back is so short that you don't feel altogether human but like an accordion ... head sinking into body."

Lukianowicz, too, reported on 23 schizophrenics with body image disorders and, although he was particularly interested in the delusions which these patients held, he emphasized that in most cases they were based on an abnormal perceptual experience. For example, one believed that he was turning into a cat because he experienced his hands and feet shrinking into cat-sized paws. Another experienced his head enlarging in size until it became as big as a lion's, into which form he then believed he had been transformed. Taylor and Fleminger reported 27 schizophrenics with what they called hypochondriacal delusions, but here too the primary psychopathology was in most cases an anomalous perceptual experience, albeit bizarre, e.g. particles of brain flying out of the left eye, a hole appearing in the centre of the left palm. In addition, these authors noticed a tendency for the delusions to be lateralized 15 left-sided, 9 right and 3 bilateral overall, but in the 10 right-handed males it was 8 left, 1 right and 1 bilateral.

A series of 100 consecutively admitted schizophrenics under my care will be reported in this article to explore further the incidence of body image disorders in schizophrenia and any particular pattern, with special reference to the five classes of body image disorders outlined above.

A final purpose of the article is then to compare the pattern of the schizophrenic body image disorders so identified with those seen following right- and left-sided brain damage. There is considerable evidence that many of the phenomena of schizophrenia arise from a neuropsychological substrate of hemisphere imbalance. The cause of this is completely unknown, but from a variety of sources of evidence-neuropsychological test results (Scarone et al., 1987), electrophysiological recordings (Morihisa $e t$ al., 1983), cerebral blood flow (Gur et al., 1985) and PET scan studies (Buchsbaum et al., 1987)-it is becoming increasingly apparent that the nature of schizophrenia can be construed in terms of hemisphere imbalance. What is contested by those who have studied the matter is the precise pattern of this hemisphere imbalance. Flor-Henry (1969) has maintained that left hemisphere hypofunction is the core problem. I have proposed (Cutting, 1985) that right hemisphere hypofunction provides the essential pathogenic influence. One way of testing each of these hypotheses is to compare a class of clinical 
phenomena which occur under conditions of left hemisphere damage and right hemisphere damage with the same class which occur in schizophrenia. If, as outlined above, body image disorders appear in all three conditions then it should be possible to examine the left-hemisphere-dysfunction and the right-hemisphere-dysfunction-hypotheses concerning schizophrenia by noting whether the body image disorders seen in schizophrenia resemble those appearing in left-hemisphere-damaged patients more than they do those of right-hemisphere-damaged patients or vice versa.

\section{Anomalous Bodily Experiences in Patients with Definite Right- and Left-Sided Brain Damage}

As mentioned above, although there is good evidence that three of the five classes of body image disorders have a particular link with damage to only one side of the brain-impaired body part identification and morbid dislike or preoccupation with left-sided, bodily unawareness with right-sided damage--the picture with respect to anomalous bodily experiences is uncertain. (False bodily experiences will not be further considered with respect to any underlying lateralized damage. Certainly autoscopic hallucinations occur almost equally with lesions of either right or left hemisphere-Cutting, 1990).

Information on the pattern of anomalous bodily experiences in left- and right-hemisphere-damaged patients was obtained from the work of two sets of authors.

The first (Table 2) is a report by Arseni et al. (1966) of 13 epileptics with anomalous bodily experiences. The most obvious finding is that the abnormally experienced body part was contralateral to the side of the lesion. It is also notable that there were no right-handers with a left-sided lesion among the sample.

TABLE 2. Arseni et al. (1966) study of 13 epileptics with anomalous bodily experiences

\footnotetext{
9 right-handers with right parietal/temporal focus

3 left hand or arm longer

1 left leg longer

1 left fingers telescoping

1 left fingers detaching

1 left arm detaching

1 left half of face longer

1 left half of tongue swollen

3 left-handers with left parietal/temporal focus

1 right hand smaller

1 right hand disappearing

1 little finger of right hand duplicated

1 right-hander with bilateral lesions both hands detaching
} 
The second source of information is a review of body image disorders by Hecaen and Ajuriaguerra, published as Méconnaissances et Hallucinations Corporelles in 1952, which is by far and away the best piece of writing on body image disorders even today. In one of the chapters the authors provide a comprehensive review of all the cases in the world literature to that date where there was a reasonably well verified unilateral lesion and an adequate clinical description of anomalous bodily experiences. There were 13 with a left-sided lesion (Table 3) and 12 with a right-sided lesion (Table 4). In rough agreement with Arseni and colleagues, the abnormally experienced body part was nearly always contralateral to the side of the lesion, and, if not, bilateral, never ipsilateral. However, there was no indication, assuming these 25 cases are representative of the true state of affairs, that right-sided lesions are more commonly the cause of anomalous bodily experiences than are left-sided lesions. A qualitative difference, however, may be discerned in the range of experiences of the left- and right-sided-damaged groups. Among the 13 with a left-sided lesion 7 experienced an absence of a body part and a further 2 a duplication, whereas neither of these themes occurred in the right-sided lesioned group, whose experiences were mainly concerned with a sense of detachment or non-belongingness of an existing body part.

So, in achieving the first purpose of this article, we can conclude that, overall, anomalous bodily experiences do not have a specific link with damage to one or other hemisphere. What does emerge, however, is that the area of the body which is the focus of the abnormal experience is nearly always contralateral to the side of the brain damage, and not once in the 38 cases reviewed here was it ipsilateral. Moreover, there is a suggestion that the quality of the anomalous experience is different depending on which hemisphere is the seat of the damage--a sense of disappearance or duplication being common in the case of left-sided damage, a sense of nonbelongingness or spatial dislocation in the case of right-sided damage.

TABLE 3. Anomalous bodily experiences in 13 patients with left-sided lesions (Hecaen and Ajuriaguerra, 1952)

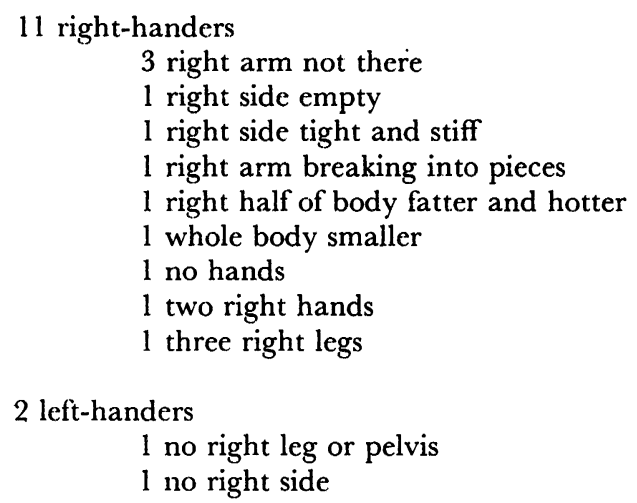


TABLE 4. Anomalous bodily experiences in 12 patients with right-sided lesions (Hecaen and Ajuriaguerra, 1952)

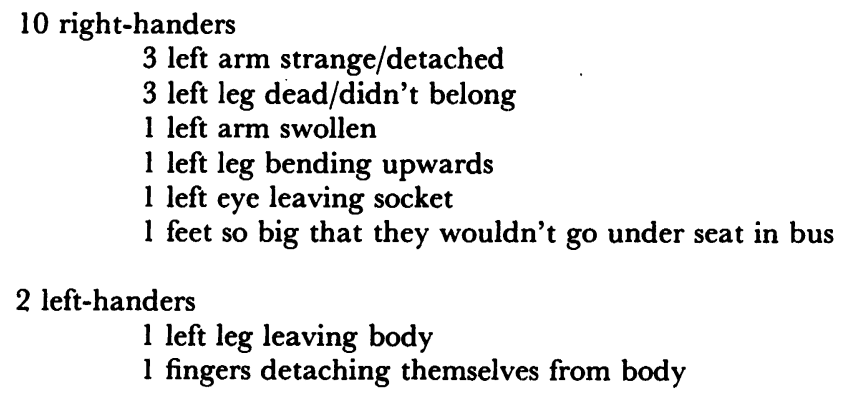

\section{Body Image Disorders in Schizophrenia}

For the purpose of identifying the incidence and range of body image disorders in schizophrenia I examined the hospital notes of the last 100 schizophrenics admitted under my care. I had seen them all personally although they had not been specifically asked about body image disorders. This will have tended to reduce the incidence of body image disorders. They all fulfilled the Research Diagnostic Criteria of Spitzer et al. (1975). They were probably not representative of the general population of schizophrenics, because about half were referred as second opinions from other psychiatrists.

Altogether 45 were recorded as having some sort of body image disorder (Table 5). I did not include subjects who only had a disorder of self-image, such as altered identity, nor did I include subjects whose abnormal beliefs about their body were restricted to notions of control or influence. I have

TABLE 5. Range of body image disorders in 100 schizophrenics

\begin{tabular}{lc}
\hline Altered structure/shape/weight & 14 \\
Tactile hallucination & 7 \\
Addition to body & 6 \\
Device present & 5 \\
Hypochondriacal & 4 \\
Physical gender change & 3 \\
Pregnant & 3 \\
Body deletion & 3 \\
Internal movement of body part & 2 \\
Body dead & 1 \\
& - \\
Lateralized body image disorder & 48 instances in 45 patients \\
& 13 left \\
& 1 right \\
& - \\
& 14 instances
\end{tabular}


subdivided the disorders in a rather arbitrary fashion into ten classes of experiences.

Altered structure, shape or weight were the commonest (facial shape different-8, hunchback-1, head stretched from body-1, skin pockmarked -1 , arms disjointed from body -1 , body lighter-1, penis turning inside out-1). Tactile hallucinations included stinging feelings, electricity, tingling and electricity in the left side, wind coming through vagina onto left side, pop star's penis in left hand, hair on fire, smacks on bottom. Six patients believed that something had been added to their body-bag of fluid, husband's girlfriend sitting on head, cat semen in blood, aeroplane on head, babies inside, two flaps of skin on abdomen with hermaphrodite penis tucked behind. Five patients believed they had a specifically localized device in their body-microphone in left ear, microcomputer in left temple, devices in ears and genitals, microchips in ears and wires on back. Four had hypochondriacal beliefs--venereal disease, thyroid disease and osteoarthritis, blood stopping, and blisters on feet and palms oozing gel. Three believed that some part of their body had been removed-left half of thyroid gland, womb, genitals.

The most surprising finding was that of the 14 instances of a lateralized abnormality 13 were left-sided.

The second purpose of the article, to record the range of body image disorders in schizophrenia, was achieved, with the main results being: (1) the incidence of these is about $50 \%$; (2) the disorder is lateralized in about onethird of instances, overwhelmingly left-sided; and (3) altered body part structure and bodily additions or deletions account for about half of the disorders.

\section{Body Image Disorders in Unilaterally-Brain-Damaged Patients and Schizophrenics Gompared}

The final purpose of this article is to use the knowledge gained about body image disorders in schizophrenia to further our knowledge about the nature of schizophrenia, with particular respect to whether left-sided or right-sided cerebral dysfunction underlies the condition.

First, the two classes of body image disorders which are most clearly linked in the neuropsychological literature with left-sided brain damageimpaired identification of body parts and a morbid preoccupation with a body part-did not feature at all in the 45 instances of schizophrenic body image disorders. Angyal (1936) also noted that, among his sample, what he called "impaired orientation within the body" was normal. Secondly, the particular themes of absence or duplication of body parts, which made up most of the instances of body image disorders in Hecaen and Ajuriaguerra's (1952) 13 patients with a left-sided lesion, did not feature among the schizophrenics either. Thirdly, the overwhelming predominance of left-sided body image disorders among schizophrenics who did have a lateralized disorder is inconsistent with left-sided brain damage in this condition as none of the 38 
patients reviewed here with a unilateral brain focus for their body image disorder had an ipsilateral disorder.

Turning now to the issue of whether there is right-sided cerebral dysfunction in schizophrenia, the findings reported or reviewed here are much more favourable. It is true that among the schizophrenics there was no gross instance of bodily unawareness, the class of body image disorders which is most closely linked with right hemisphere damage, although there were a handful of cases with the experience of body part deletions, including one man who believed that the left part of his thyroid gland had disappeared. However, the themes which were identified in those with schizophrenic body image disorders were generally in keeping with the themes uncovered in the two reports of patients with right-sided-damage-induced body image disorders. Altered bodily structure was the commonest theme in schizophrenics, in essence a sense that a body part, although recognized as existing, was different in its spatial location. Consider this schizophrenic man, not in the present series:

"I felt that all my arms and legs had been thrown into a bag. I knew that they were acting according to my will, but they were somehow displaced from their normal position. My arm seemed to be sticking out of my chest. My mouth was where my hair should be. And the two lobes of my brain were revolving round and round; maybe they were crossed over, almost as if they has reversed themselves."

It is clear that the normal arrangement of his body parts, their normal spatial relationships, one to another, had been disrupted. This same theme was common in those with a right hemisphere lesion, where body parts were experienced as detaching from their customary spatial position. Most of all, the left-sided predominance of the body image disorders in the schizophrenics is, according to all the evidence considered in this article, strongly in favour of the presence of right-sided cerebral dysfunction in the condition. The consistent link between a left-sided abnormal bodily experience and a right-sided cerebral lesion, observed in the 38 cases of unilateral brain lesions reviewed in this article, testifies to the fact that leftsided body image disorders arise out of right-sided cerebral dysfunction.

In conclusion, therefore, a comparison between patients with left-sided brain lesions, right-sided brain lesions and those with schizophrenia in terms of the body image disorders which they develop, yields the conclusion that, in this respect, schizophrenics overwhelmingly resemble patients with rightsided lesions more then they do those with left-sided lesions.

\section{References}

Angyal, A. (1936). The experience of the body-self in schizophrenia. Archives of Neurology and Psychiatry, 35, 1029-1053.

Arseni, C., Botez, M. I. and Maretsis, M. (1966). Paroxysmal disorders of the body image. Psychiatria et Neurologia, 151, 1-14.

Babinski, M. J. (1914). Contribution à l'étude des troubles mentaux dans l'hémiplégie organique cérébrale. Revue Neurologique, i, 845-848.

Buchsbaum, M. S. et al. (1987). Positron emission tomography studies of basal ganglia and somatosensory cortex neuroleptic drug effects. Biological Psychiatry, 22, 479-494. 
Critchley, M. (1951). Types of visual perseveration: "paliopsia" and "illusory visual spread". Brain, 74, 267-299.

Critchley, M. (1979). Misoplegia or hatred of hemiplegia. In "The Divine Banquet of the Brain", Raven, New York, pp. 115-120.

Cutting, J. (1978). Study of anosognosia. Fournal of Neurology, Neurosurgery and Psychiatry, 41, $548-555$.

Cutting, J. (1985). "The Psychology of Schizophrenia". Churchill Livingstone, Edinburgh.

Cutting, J. (1990). “The Right Cerebral Hemisphere and Psychiatric Disorders". Oxford University Press, Oxford.

De Renzi, E. and Scotti, G. (1970). Autotopagnosia: fiction or reality? Archives of Neurology, 23, 221-227.

Flor-Henry, P. (1969). Psychosis and temporal lobe epilepsy: a controlled investigation. Epilepsia, 10, 363-395.

Gooddy, W. and Reinhold, M. (1953). Some aspects of human orientation in space. II The dynamic nature of nervous activity. Brain, 76, 337--363.

Gur, R. E. et al. (1985). Brain function in psychiatric disorders. III Regional cerebral blood flow in unmedicated schizophrenics. Archives of General Psychiatry, 42, 329-334.

Head, H. and Holmes, G. (1911). Sensory disturbances from cerebral lesions. Brain, 34, 102254.

Hecaen, H. and Ajuriaguerra, J. de (1952). "Méconnaissances et Hallucinations Corporelles." Masson, Paris.

Hecaen, H. and Sauguet, J. (1971). Cerebral dominance in left-handed subjects. Cortex, 7, $19-48$.

Lukianowicz, N. (1967). Body image disturbances in psychiatric disorders. British fournal of Psychiatry, 113, 31-47.

Lunn, V. (1948). "Om Legernsbevidstheden Belyst ved Nogle Forstyrrelser of den Normale Oplevelsesmaade". Munksgaard, Copenhagen.

Morihisa, J. M., Duffy, F. H. and Wyatt, R. J. (1983). Brain electrical activity mapping in schizophrenic patients. Archives of General Psychiatry, 40, 719-728.

Ogden, J. A. (1985). Autotopagnosia, Brain, 108, 1009-1022.

Scarone, S., Cazzullo, C. L. and Gambini, O. (1987). Asymmetry of lateralised hemispheric functions in schizophrenia. Influence of clinical and epidemiological characteristics on quality extinction test performance. British Journal of Psychiatry, 151, 15-17.

Schilder, P. (1935). "The Image and Appearance of the Human Body." Kegan Paul, Trench and Trubner, London.

Spitzer, R. L., Endicott, J. and Robins, E. (1975). "Research Diagnostic Criteria". New York State Psychiatric Institute, New York.

Stengel, E. (1944). Loss of spatial orientation, constructional apraxia and Gerstmann's syndrome. Fournal of Mental Science, 90, 753-766.

Taylor, P. and Fleminger, J. J. (1981). The lateralization of symptoms in schizophrenia. British Fournal of Medical Psychology, 54, 59-65.

Van Bogaert, L. (1934). Sur la pathologie de l'image de soi. Annales Médicopsychologiques, 92, 519-555, 744-759. 


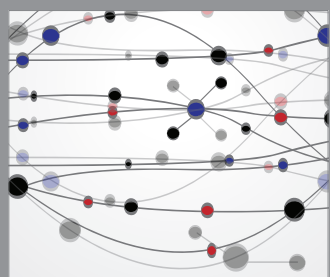

The Scientific World Journal
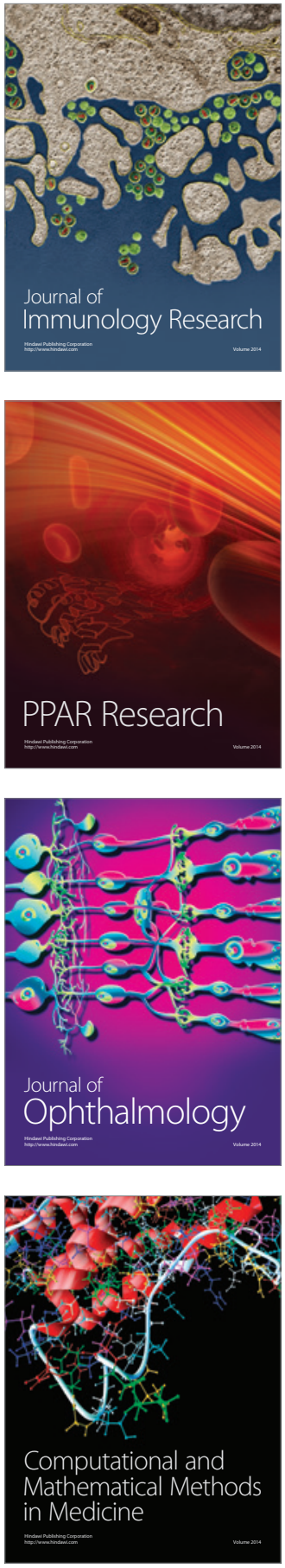

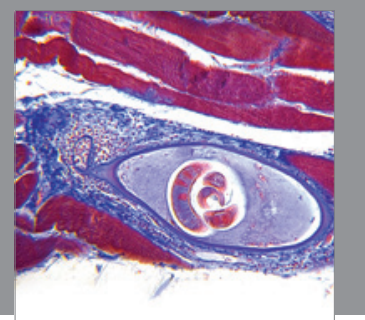

Gastroenterology

Research and Practice
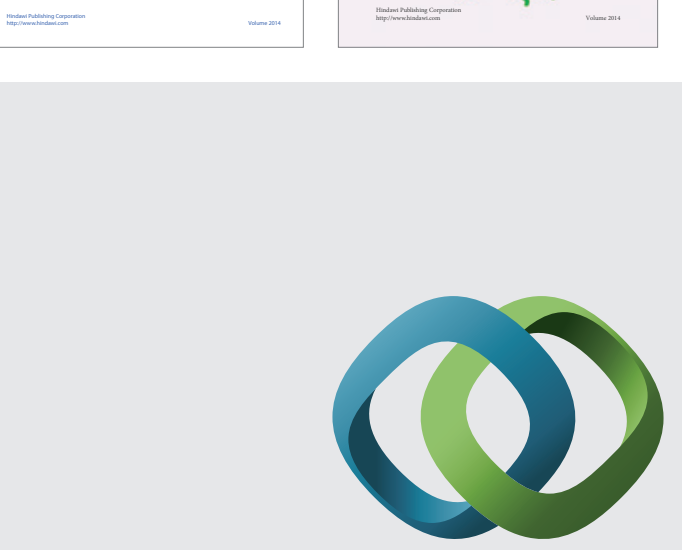

\section{Hindawi}

Submit your manuscripts at

http://www.hindawi.com
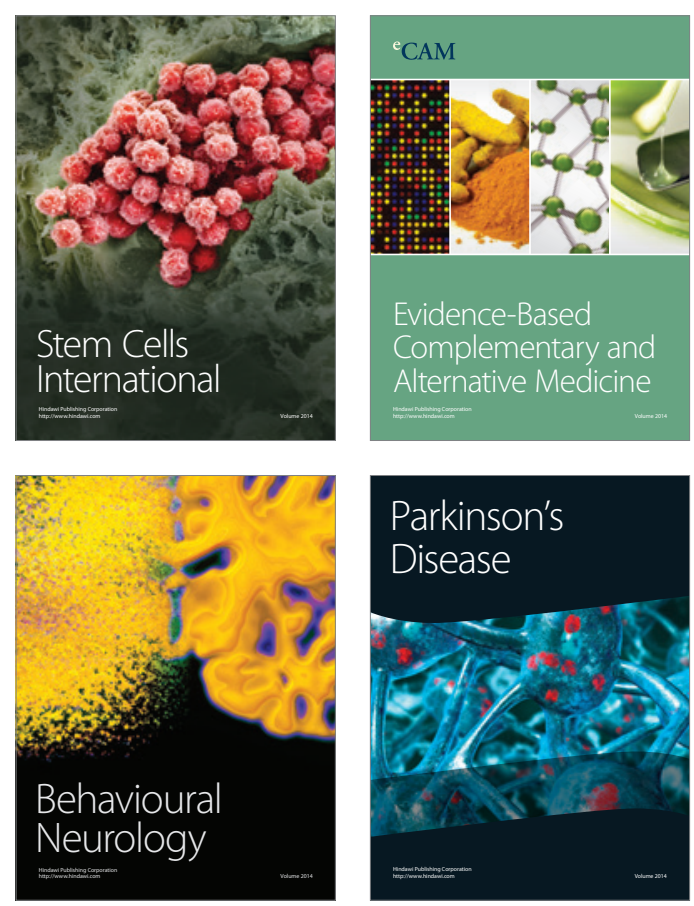

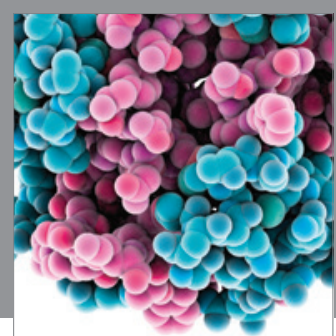

Journal of
Diabetes Research

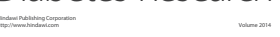

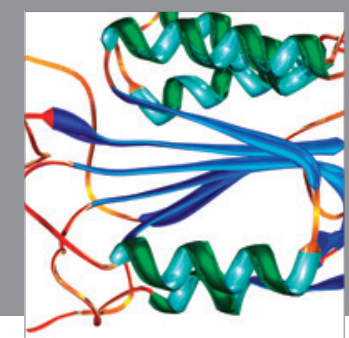

Disease Markers
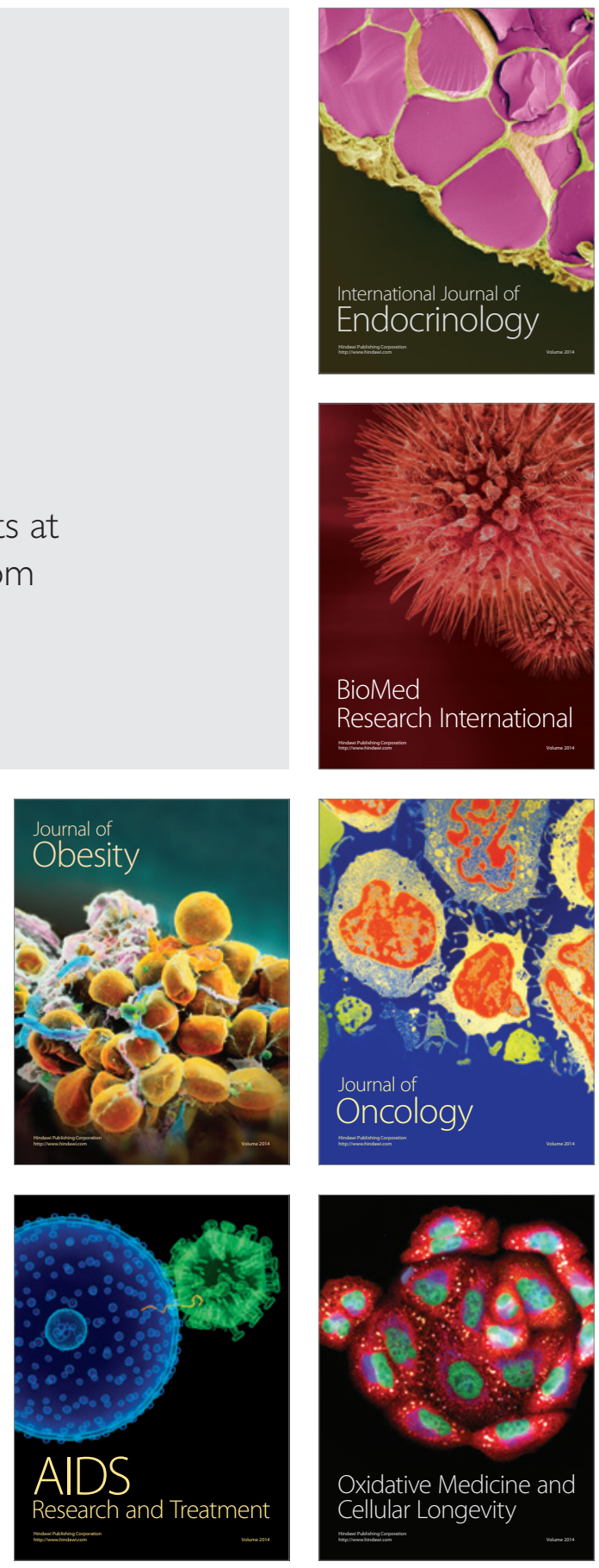